\title{
Cue utilization and hippocampal lesions in rats
}

\author{
FREDERICK G. FREEMAN \\ Old Dominion University, Norfolk, Virginia 23508
}

\begin{abstract}
Hippocampal lesioned and sham-operated control rats were trained on a go, no-go task to assess the importance of multiple relevant cues on learning. When one stimulus (e.g., a light) was presented during the go trials and a second stimulus (e.g., a tone) was presented during the no-go trials, the lesioned animals performed more poorly than the control animals. However, when a light-tone combination was presented during the go (or no-go in a second condition) trials, and their absence signified the no-go trials (or the go trials), the performance of the lesioned and control animals did not differ. The data suggest that the use of multiple cues in an operant go, no-go discrimination task does not necessarily eliminate the learning deficit typically seen on this task by animals with hippocampal lesions. The manner in which these cues are presented is also important.
\end{abstract}

Lesions of the hippocampus have produced behavioral deficits on a variety of learning tasks (cf. Douglas, 1967; Kimble, 1968). However, many investigators have reported that increasing the number of relevant cues in behavioral tasks will enhance the performance of animals with hippocampal lesions to the point that their behavior does not differ from that of control animals. On such divergent tasks as maze learning (Winocur \& Breckinridge, 1973), DRL schedules (Pelligrino \& Clapp, 1971; Rickert, Bennett, Anderson, \& Corbett, 1973), and single alternation (Stevens \& Cowey, 1972), the adding of relevant cues has facilitated learning by lesioned animals.

The present research examined the effects of multiple cues on acquisition of a go, no-go operant discrimination task by animals with hippocampal lesions. Although a number of investigators have reported learning deficits on such a task under a variety of conditions (e.g., Freeman \& Kramarcy, 1974; Freeman, Kramarcy, \& Lee, 1973; Schmaltz, Wolf \& Trejo, 1973; Swanson \& Isaacson, 1967; Woodruff, Means \& Isaacson, 1973), in each of these studies only one relevant cue was provided for the learning of the task. The purpose of the present study was to examine the effect of presenting several relevant stimuli for the learning of a go, no-go discrimination task by rats with hippocampal lesions. Since the period in which a stimulus is presented may also be an important variable (cf. Freeman et al., 1973), the effect of presenting the relevant stimuli during either the go or the no-go, or during both of these periods, was also examined.

\section{EXPERIMENT 1}

\section{Method}

Subjects. The subjects were 20 male Long-Evans rats, approximately 90-120 days old at the time of the operation. All animals

This research was supported by a summer grant from the Old Dominion University Research Foundation. were individually housed and maintained on a 12-h light-dark cycle.

Apparatus. All animals were trained in sound-attenuated operant conditioning chambers. A retractable lever was situated in the middle of one wall of the chamber $3 \mathrm{~cm}$ above the grid floor. A speaker from which tones could be presented was situated in the ceiling $20 \mathrm{~cm}$ directly over the lever. A cue light was located on the same wall as the lever and $8 \mathrm{~cm}$ above the lever. The pellet dispenser was situated $3 \mathrm{~cm}$ directly to the left of the response lever. Reinforcement consisted of a 45-mg Noyes pellet. A bulb in the rear of the chamber served as a houselight.

Surgery. All surgery was performed under sodium pentobarbital anesthesia (50 mg Nembutal/kg body weight). Ten animals received two bilateral lesions of the hippocampus. With the incisor bar set $5 \mathrm{~mm}$ above the interaural plane and using bregma as the zero point, the following coordinates were employed: posterior, $2.5 \mathrm{~mm}$; lateral, $\pm 2.5 \mathrm{~mm}$; ventral, $3.2 \mathrm{~mm}$; and posterior, $3.8 \mathrm{~mm}$; lateral, $\pm 4.3 \mathrm{~mm}$; ventral $4.3 \mathrm{~mm}$. Lesions were produced electrolytically by passing a $2-\mathrm{mA}$ anodal current through the uninsulated $.5 \mathrm{~mm}$ tip of a stainless steel electrode for $20 \mathrm{sec}$. The remaining 10 animals received a sham operation in which they were anesthetized, placed in the stereotaxic instrument, and had an incision made in their scalps. All animals were allowed to recuperate from the operation for 10 days prior to the initiation of barpress training. On the 8th day of recuperation, all animals were put on a food-deprivation schedule and maintained at $85 \%$ of their ad-lib weights for the remainder of the experiment.

Procedure. On Day 1 of training, all animals were shaped to barpress. Once responding was begun, the animals were allowed to press for 50 reinforcements on a continuous schedule (CRF) each day for 5 days. On the first 2 days, the bar always remained in the chamber. On Days 3-5, the bar remained in the chamber for $10 \mathrm{sec}$ (one trial) and was removed for a $20 \mathrm{sec}$ intertrial interval (ITI). Neither the tone nor the cue light to be used as discriminanda were present during the first 5 days. The houselight came on whenever the lever was presented to the animal. Intersession intervals were approximately $23 \mathrm{~h}$. On Day 6, discrimination training began. For five of the lesioned (HL+T-) and five of the control animals (SL+T-), responses were rewarded on a CRF schedule on trials when the cue light was on and not rewarded when a $1,500-\mathrm{Hz}, 85-\mathrm{dB}$ tone $\mathrm{S}+$ (re .0002 dynes $/ \mathrm{cm}^{2}$ ) was presented. For the remaining lesioned animals $\left(\mathrm{HT}+\mathrm{L}_{-}\right)$and control $\left(\mathrm{ST}+\mathrm{L}_{-}\right)$animals in each group, responses made on trials when the tone was presented $(\mathrm{S}+)$ were reinforced while responses made in the presence of the light $\left(\mathrm{S}_{-}\right)$ were extinguished. Ambient noise level in the experimental chambers was approximately $55 \mathrm{dBA}$. Fifty $\mathrm{S}+$ and $50 \mathrm{~S}-$ 
trials were randomly presented each day. The houselight was presented only when a trial was in progress. All animals were trained for 5 consecutive days.

On Day 11, each animal was given a generalization test. The test began with a warm-up period of $25 \mathrm{~S}+$ and $25 \mathrm{~S}$ - trials randomly presented, as in training. Following this, the following stimuli were presented to each animal: the tone-light combination, the tone alone, the light alone, or nothing. Eight series of these stimuli were presented with one presentation of all stimuli constituting a series. The stimuli were presented in trials just as in training, with the presentation of each stimulus randomized within a series. No responses were reinforced during the generalization test.

Histology. At the end of the experiment, all animals were injected with an overdose of Nembutal and perfused with $.9 \%$ saline followed by $10 \%$ Formalin solution. The brains were removed and stored in $10 \%$ Formalin. Frozen sections were cut at a 50- $\mu$ thickness and stained with cresyl violet.

\section{Results}

Damage to the dorsal hippocampus was observed in all of the lesioned animals. The lesion typically extended posteriorventrally with some destruction of the ventral hippocampus. In addition, in some animals, the cortex and dorsal thalamus and lateral geniculate were partially damaged. The minimum and maximum extent of the lesions are provided in Figure 1.

The bottom half of Figure 2 presents the percent of total responses made to the $\mathrm{S}+$ over the 5 days of training. Examination of this figure suggests that Group ST+L- performed better than all other groups; Group HL+T - appears to have the poorest performance; and Groups $\mathrm{HT}+\mathrm{L}-$ and $\mathrm{SL}+\mathrm{T}-$ appear to have performed at an intermediate level. A 2 by 2 by 5 analysis of variance performed on the percent of total responses made to the $\mathrm{S}+$ across days yielded a significant lesion effect $[F(1,16)=6.0, p<.05]$ and a significant stimulus condition effect $[F(1,16)=5.32$, $\mathrm{p}<.05]$. However, none of the interactions were significant. Thus, the sham-operated animals performed

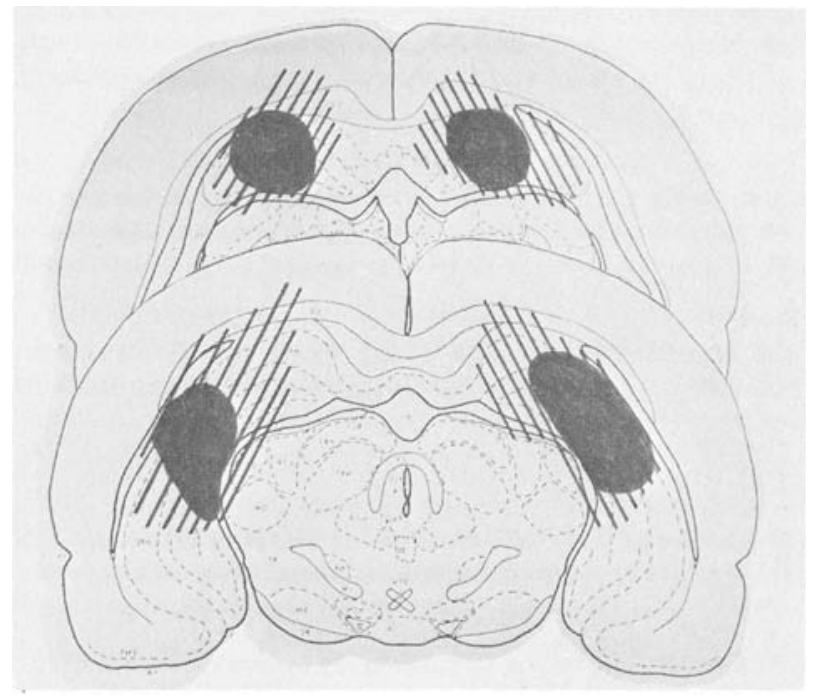

Figure 1. Minimum (dark) and maximum (striped) extent of hippocampal lesions.

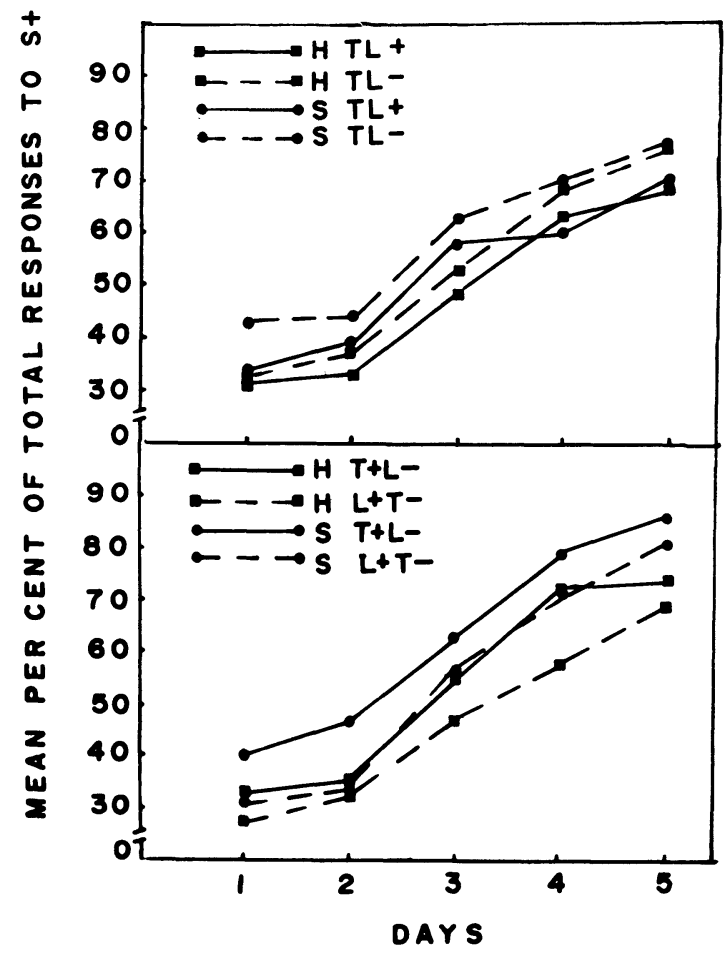

Figure 2. Percent of total responses made to the $S$-stimulus for Experiment 1 and Experiment 2 (top).

significantly better than the lesioned animals and the $\mathrm{T}+\mathrm{L}-$ animals performed significantly better than the $\mathrm{L}+\mathrm{T}-$ animals. The lack of a significant Lesion by Stimulus Condition interaction $(\mathrm{F}<1.0)$ precluded any other group comparisons.

An analysis of S+ response rates did not yield any significant effects except for an increase in responding for all groups across days $[F(4,64)=29.55, p<.01]$. An analysis of responses to the $\mathrm{S}$ - stimulus across days showed that the lesioned animals responded significantly more than control animals $[F(1,16)=5.56, p<.05]$. Animals in the $\mathrm{L}+\mathrm{T}$ - stimulus condition tended to respond more to the $\mathrm{S}-$ than those in the $\mathrm{T}+\mathrm{L}-$ condition across days, but this effect failed to reach statistical significance. Also, there was a significant decrease in $\mathrm{S}-$ responses across days $[\mathrm{F}(4,64)=29.46, \mathrm{p}<.01]$ but no significant interactions between any of the variables.

Table 1 presents the absolute number of responses made to each stimulus during the generalization test. There was a significant test stimulus effect $[F(3,48)=$ 2.98, $\mathrm{p}<.05$ ] and a significant Test Stimulus by Stimulus Condition interaction $[\mathrm{F}(3,48)=29.79$, $\mathrm{p}<.01]$. These effects are attributable primarily to the $\mathrm{L}+\mathrm{T}-$ groups responding most to the light and least to the tone during the test, with the reverse being true for the $\mathrm{T}+\mathrm{L}-$ groups. The intermediate level of responding by three of the groups in the "nothing" test condition is presumably due to generalization decrement. That is, the stimulus condition involving the absence of both 
Table 1

Mean Number of Responses Made to Each Stimulus During the Generalization Test for Experiment 1

\begin{tabular}{llrrrr}
\hline & & Tone- & & & \\
& & Light & Tone & Light & Nothing \\
\hline \multirow{2}{*}{ Hippocampal } & T+L- & 8.6 & 24.8 & 1.2 & 9.6 \\
& L+T- & 15.0 & 6.4 & 18.8 & 10.2 \\
\multirow{3}{*}{ Sham } & T+L- & 4.4 & 25.4 & 2.4 & 14.6 \\
& L+T- & 10.0 & 1.2 & 17.6 & .4 \\
\hline
\end{tabular}

stimuli may be presumed to lie on a hypothetical continuum between the presence of either the $\mathrm{S}+$ or $\mathrm{S}-$ stimuli.

\section{Discussion}

The learning deficits in rats with hippocampal lesions suggests that using several relevant stimuli in a go, no-go discrimination task does not necessarily enhance performance. The finding that the two hippocampal lesion groups performed at significantly lower levels than their respective control groups replicates earlier studies in which go, no-go learning deficits were found. Interestingly, the manner in which these cues were used in the present research was important in determining the deficit observed. That is, the $\mathrm{T}+\mathrm{L}$ - groups performed better than the L+T-groups. The reason for this effect is not clear. Conceivably, one of the cues provided more information value when presented during either the go or no-go trials than when presented during the opposite period. An earlier study by Freeman et al. (1973), which used a similar procedure, provides some support for this notion. In that study, when a tone was presented during the no-go periods, animals with hippocampal lesions took significantly longer than control animals to learn. However, when the tone served as the go cue, no such deficit was found. Perhaps, in the present task, the lesioned animals were not utilizing the tone cue, presented during no-go trials, as well as control animals. If this were true, the task could have involved only one "functional" stimulus for the lesioned subjects. Although this explanation may account for the learning deficit of the lesioned animals, the generalization data do not suggest that there were any significant diffe rences in the manner by which cues were utilized in learning the task.

In an attempt to examine further the importance of several relevant stimuli in a go, no-go discrimination task, a second experiment was conducted in which two cues were presented together during either the $S+$ or $\mathrm{S}-$ period. If animals with hippocampal lesions do have difficulty learning about cues associated with nonreinforcement, their performance should be markedly deficient when the relevant cues are presented only during the $\mathbf{S}-$. On the other hand, if increasing the number of relevant cues facilitates learning by rats with hippocampal lesions (e.g., by increasing the discriminability of the go and no-go intervals), the deficit in these animals could be reduced.

\section{EXPERIMENT 2}

\section{Method}

Subjects. The subjects consisted of 20 male Long-Evans rats, approximately 90 days of age at the time of the operation.

Procedure. All surgical, postoperative care, discrimination training, and generalization testing procedures were the same as in Experiment 1, with the following exception. For five of the lesioned and five of the control animals, the tonc and light were presented during the $\mathrm{S}+$ period and absent during the $\mathrm{S}-$ period (TL+). For the remaining animals in each group, the light and tone were presented during the $\mathrm{S}-$ period and absent during the S+ (TL-).

\section{Results}

Histological examination revealed essentially the same results reported in Experiment 1.

No effect on the discrimination performance by either the lesion or the stimulus condition variables was observed. The percent of total responses made to the $\mathrm{S}+$ stimulus on Days 1-5 is presented in the top panel of Figure 2. Although Group HTL+ appeared to be performing at a lower level than the other three groups on Day 5, an analysis of variance of the percent of total responses made to the $S+$ across the 5 days of training did not yield a lesion, a stimulus condition, or any interaction effects (all Fs $<1.0$ ). An analysis of $S+$ response rates also did not yield any significant differences between groups (all Fs $<1.0$ ). There was a significant increase in responding in the presence of the S+ across days for all groups $[F(4,64)=25.96, p<.001]$. An analysis of $S$ - response rates failed to yield either a lesion or a stimulus condition effect, but revealed a significant Lesion by Days interaction $[F(4,64)=2.82$, $\mathrm{p}<.05]$. Newman-Keuls comparisons showed that the lesioned animals made significantly more $\mathrm{S}-$ responses on Days 1 and $2(p<.05)$ but did not differ from the control subjects on the last 3 days.

Table 2 presents the mean number of responses made to each stimulus during the generalization test. The Training Condition by Test Stimulus interaction was significant $[F(3,48)=43.04, p<.01]$; this reflected the differential responding to the test stimuli as a function of training regardless of the lesion condition. Animals with hippocampal lesions tended to make more responses to the test stimuli during the test than the

Table 2

Mean Number of Responses Made to Each Stimulus During the Generalization Test for Experiment 2

\begin{tabular}{llrrrr}
\hline \multirow{5}{*}{ Hippocampal } & \multicolumn{5}{c}{ Tone- } \\
Light & Tone & Light & Nothing \\
\hline \multirow{3}{*}{ Sham } & TL+ & 32.8 & 7.4 & 23.8 & 4.6 \\
& TL- & 6.2 & 10.6 & 15.2 & 25.4 \\
& TL+ & 20.2 & 10.0 & 13.2 & 3.6 \\
& TL- & 3.8 & 16.4 & 5.2 & 22.2 \\
\hline
\end{tabular}


control animals $[\mathrm{F}(1,16)=3.54, \mathrm{p}<.10]$. In addition, there was a significant Lesion by Stimulus Condition interaction $[F(3,48)=5.25, p<.01] \quad$ Newman-Keuls comparisons revealed that the lesioned animals tended to respond significantly more to the light $(p<.01)$ than the control subjects and less to the tone, although this latter tendency was not significant. Relative gradients in which the percent of total responses to each stimulus were obtained yielded the same results.

\section{DISCUSSION}

The results of Experiment 1 are compatible with earlier studies in which a learning deficit on a go, no-go operant discrimination was found in animals with hippocampal lesions. The results of Experiment 2 are compatible with previous studies, using a variety of tasks, in which it has been found that increasing the number of relevant cues facilitated the performance of hippocampal lesioned animals to the point where they did not differ significantly from nonlesioned control animals. The results of Experiments 1 and 2 taken together, however, suggest that the manner in which relevant cues are presented in a go, no-go task is an important variable in affecting the learning of animals with hippocampal lesions.

It was hoped that the generalization tests would provide information concerning the manner in which cues were utilized by the various groups in learning the discrimination task. The results of these tests, however, yielded somewhat ambiguous results. For example, it is not clear why the lesioned animals responded more to the light in the generalization test of Experiment 2. Conceivably, animals in the TL+ condition were using the light as the cue for learning the discrimination task, as reflected in the generalization test. In the TL- condition, however, the sham subjects appeared to be using the light more than the tone as a cue not to respond as reflected by the generalization test. The lesioned subjects, however, appeared to rely on the presence of both cues. Thus, when the light alone was present during the test, the lesioned subjects tended to respond more, accounting for their overall greater response rate to the light. Presumably, the combination of the tone and light during the $\mathrm{S}$ - period of discrimination learning formed a compound stimulus for the lesioned subjects, making the $S+$ and $S$ - periods more discriminable, thus facilitating learning. Regardless of the mechanism by which such responding occurred, the results do suggest that the animals with hippocampal lesions did not utilize the relevant cues in the same manner as the control animals. This cannot be attributed to a general tendency of the lesioned animals elicited by some idiosyncracy of the discrimination paradigm employed, as no such tendency to increase responding to the light was observed in Experiment 1.
In Experiment 1, where only one stimulus was presented during the go and no-go trials, the two periods may have been less discriminable (cf. Woodruff et al., 1973). Although the results of the generalization test did not provide support for such a hypothesis, it would seem to be the most plausible explanation, especially when considered in conjunction with previous investigations (e.g., Winocur \& Breckinridge, 1973). Perhaps other paradigms, such as transfer of training, might have provided a more clear-cut assessment of how the various groups utilized the various cues.

In order to test the latter hypothesis, that the distinctiveness of the go and no-go periods is the critical factor, such variables as the intensity of the discriminative stimuli might be manipulated as suggested by Woodruff et al. (1973). With a less intense stimulus, the lesioned animal might be less likely to "notice" the cue, and thus show a learning deficit. With a more intense stimulus, or with several stimuli, as in the present experiment, the two periods would become more discriminable and the deficit in animals with hippocampal lesions decreased.

\section{REFERENCES}

Douglas, R. J. The hippocampus and behavior. Psychological Bulletin, 1967, 67, 416-442.

Freeman, F. G., \& Kramarcy, N. R. Stimulus control of behavior and limbic lesions in rats. Physiology \& Behavior, 1974, 13, 609-615.

Freeman, F. G., Kramarcy, N. R., \& Lee, J. Discrimination learning and stimulus generalization in rats with hippocampal lesions. Physiology \& Behavior, 1973, 11, 273-275.

Kimble, D. P. Hippocampus and internal inhibition. Psychological Bulletin, 1968, 70, 285-295.

Pellegrino, L. J., \& Clapp, D. F. Limbic lesions and externally cued DRL performance. Physiology \& Behavior, 1971, 7, 863-869.

Rickert, E. J., Bennett, T. L., Anderson, G. J., Corbett, J., \& SMITH, L. Differential performance of hippocampally ablated rats on nondiscriminated and discriminated DRL schedules. Behavioral Biology, 1973, 8, 597-605.

Schmaltz, L. W., Wolf, B. P., \& Trejo, W. R. FR, DRL, and discrimination learning in rats following aspiration lesions and penicillin injection into hippocampus. Physiology \& Behavior, 1973, 11, 17-22.

STEvens, R., \& Cowey, A. Enhanced alternation learning in hippocampectomized rats by means of added light cues. Brain Research, 1972, 46, 1-22.

Swanson, A. M., \& IsaAcson, R. L. Hippocampal ablation and performance during withdrawal of reinforcement. Journal of Comparative and Physiological Psychology, 1967, 64, 30-35.

Winocur, G., \& Breckinridge, C. B. Cue dependent behavior of hippocampally damaged rats in a complex maze. Journal of Comparative and Physiology Psychology, 1973, 82, 512-532.

Woodruff, M. L., Means, L. W., \& IsaAcson, R. L. Deficient go, no-go brightness discrimination in rats following hippocampal lesions. Physiological Psychology, 1973, 1, 85-88.

(Received for publication October 28, 1977; revision accepted June 20,1978 .) 